\title{
Disposición al aprendizaje y convivencia democrática en escuelas públicas del Perú
}

Disposition to learning and democratic coexistence in public schools of Peru

Mg. Carola Yamanija,

Dan Omura y

Mg. Roberto Barrientos,

Comunidades de aprendizaje

Recepción 5/ 06/2018

Revisado 9/06/2018

Aceptación 23/08/2018

\section{Resumen}

T a presente investigación busca identificar la relación entre la disposición al aprendizaje y la convivencia democrática en los estudiantes en los que se implementa el proyecto Comunidades de Aprendizaje. Asimismo, indaga si existen diferencias significativas para estas mismas variables en muestras independientes que consideran factores demográficos. Es una investigación cuantitativa, de diseño no experimental, descriptivo correlacional. En total se encuestaron a 9,944 estudiantes. Esta muestra es censal, ya que, participaron todos los estudiantes de tercer grado de primaria a quinto grado de secundaria a quienes se aplican las Tertulias Literarias en las 43 escuelas del proyecto ubicadas en Lima, Callao, Piura, Cusco, Apurímac, Huancavelica y Puno, en zonas urbanas y rurales. El instrumento aplicado fue diseñado específicamente para esta medición y sirve como línea de base para las escuelas que implementan el proyecto. Los resultados descriptivos muestran principalmente niveles altos y muy altos para la disposición al aprendizaje y la convivencia democrática. Estas dos variables presentan correlación positiva considerable $(0,678)$ y altamente significativa $(0,01)$ y al analizarlas en muestras independientes se presentaron diferencias estadísticamente significativas. Como conclusión los resultados más altos se hallan en las estudiantes mujeres, en nivel primaria y el ámbito rural. 
Palabras clave: Convivencia democrática, disposición al aprendizaje, comunidades de aprendizaje, tertulias literarias, aprendizaje dialógico

\begin{abstract}
7 his research seeks to identify the relationship between the disposition to learn and democratic coexistence in students of schools that implement

"Comunidades de Aprendizaje". It also investigates whether there are significant differences for these same variables in independent samples that consider demographic factors. It is a quantitative research, with nonexperimental, correlational descriptive design. A total of 9944 students answered this censal survey, participating all students, from third grade of primary to fifth grade of high school engaged in literary circles in any of the 43 schools of the project located in Lima, Callao, Piura, Cusco, Apurímac, Huancavelica and Puno, both in urban and rural areas. The applied instrument was specifically designed for this research and serves as a baseline for the schools that implement the project. The descriptive results show high and very high levels for the disposition to learn and democratic coexistence. These two variables show considerable and highly significant $(0.01)$ positive correlation (0.678) and when analyzing them in independent samples, statistically significant differences were found concluding that the highest results are found in female students, at the primary level and in rural areas.
\end{abstract}

Key words: Democratic coexistance, disposition to learn, learning communities, literary gatherings, dialogic learning 


\section{Introducción}

Diversas investigaciones en educación indican que existe una correlación entre la convivencia y el aprendizaje (Viñas, 2004; Jares, 2006; Elboj, Puigdellivol, Soler y Valls, 2006; LLECE, 2008; Román y Murillo, 2011; Fierro y Fortoul, 2013; Díaz y Sime, 2016). El aprendizaje en la escuela, no se desarrolla de manera aislada, se da dentro de un contexto de interacción que puede ser entre docente - alumno, alumno - alumno, docente - docente, etc. Este intercambio no es solo cognitivo, sino también se combinan emociones, valoraciones, en un entretejido de relaciones. Para Fierro y Fortoul (2013) la experiencia educativa ocurre mediante el encuentro intersubjetivo entre personas y si esta experiencia no se desarrolla bajo parámetros de bienestar psicológico, éticos y emocionales, puede volver difícil o quizá imposible enseñar y aprender, resaltando así la relación entre convivencia y aprendizaje.

Ubicándonos en un contexto social mayor, con el fenómeno de la globalización, el intercambio y el flujo migratorio, las sociedades presentan una serie de desafíos, dado el incremento de ciudadanos de distintas culturas en un mismo entorno, como el caso de Estados Unidos, Reino Unido, Emiratos Árabes, Canadá, etc. (BBC, 2017). Estos desafíos deben ser atendidos para evitar brechas, violencia social y más bien generar inclusión y sociedades más cohesionadas. Actualmente los sistemas escolares presentan este desafío global, de contemplar una educación cada vez más inclusiva, que favorezca el aprendizaje de todos, como bien señala la UNESCO, transformando las escuelas y entornos de aprendizaje para responder a la diversidad (UNESCO, 2008, p. 12).

Por ello, la importancia de favorecer la convivencia para el aprendizaje. En el contexto peruano existen algunos estudios como el de Román y Murillo (2011), que investiga la correlación entre violencia y desempeño escolar. Estos autores correlacionan el nivel de violencia con el aprendizaje tomando como base los resultados del Segundo Estudio Regional Comparativo y Explicativo (SERCE) de la Organización de las Naciones Unidas para la Educación, la Ciencia y la Cultura (UNESCO). Los autores encontraron que "un estudiante de primaria en América Latina que señala haber sido robado o maltratado física o verbalmente tiene un desempeño significativamente inferior, en lectura y matemáticas, que otro estudiante que no lo ha sido" (p. 47). De igual forma, los autores mencionan que si un estudiante asiste a un aula donde se presenta una mayor proporción de actos de robo o maltrato, este 
tiene desempeños más bajos en lenguaje y en matemáticas que un estudiante que asiste a un aula donde se presenta menor violencia, haciéndose notoria la correlación entre convivencia y aprendizaje.

Por otra parte, las escuelas y las aulas actuales tanto en el Perú como en la región, presentan una mayor diversidad cultural, lo cual es un reto para la gestión y el aprendizaje en las instituciones educativas (Vergara y Gil, 2010). Además de la diversidad cultural en el Perú, la educación rural merece atención especial; los resultados 2016 de la evaluación censal de estudiantes (ECE) muestran diferencias muy notorias entre las instituciones educativas urbanas y rurales, estas últimas siempre presentan resultados significativamente más bajos. En lectura, esta diferencia es de $34.4 \%$ en segundo de primaria; $23.2 \%$, en cuarto de primaria; y $13.8 \%$, en segundo de secundaria. En el caso de matemática, esta diferencia es de $19.3 \%$ en segundo de primaria; $15 \%$, en cuarto de primaria y $10.2 \%$, en segundo de secundaria (Unidad de la Medición de la Calidad, 2016).

"Comunidades de Aprendizaje" es un proyecto de transformación de la escuela y su entorno, que busca ofrecer a las escuelas públicas, herramientas y estrategias para mejorar la convivencia, los aprendizajes y la participación de la comunidad. A pesar de los diversos estudios y publicaciones sobre "Comunidades de Aprendizaje", no existen precedentes de un estudio sistemático, cuantitativo y correlacional considerando los principios del aprendizaje dialógico.

La pregunta principal a la que se quiso responder con este estudio fue la siguiente: ¿cuál es la relación entre la disposición al aprendizaje y la convivencia democrática en los estudiantes de $3^{\circ}$ de Primaria a $5^{\circ}$ de Secundaria de la red de escuelas que participan del proyecto "Comunidades de Aprendizaje" en el Perú?

Este estudio aporta a las investigaciones sobre la correlación entre convivencia democrática y disposición al aprendizaje en un contexto local como el Perú. A su vez, aporta en la profundización del desarrollo de este nuevo enfoque de aprendizaje llamado "dialógico". Por otro lado, la información resulta útil para las entidades gubernamentales que vienen implementando dicho proyecto y para aquellas interesadas en poner en práctica el mismo. 
Se está proponiendo una investigación cuantitativa dado que son muy escasas las investigaciones de este tipo dentro del ámbito de "Comunidades de Aprendizaje". La mayor parte de las investigaciones realizadas en Europa, sobre este proyecto, son de naturaleza cualitativa. La investigación a desarrollar es de diseño correlacional, buscando establecer la relación entre los dos focos principales del proyecto: convivencia democrática y disposición al aprendizaje, estableciéndose como una línea de base para el proyecto en Perú.

\subsection{Antecedentes}

El proyecto "Comunidades de Aprendizaje" tiene su origen en España y surge como fruto de la confluencia de aportes de diferentes áreas de investigación como la didáctica, teoría de la educación, la sociología, el estudio del desarrollo, etc., principalmente las líneas de investigación desarrollado por el CREA de la Universidad de Barcelona (Elboj, et.al, 2016). Dado que es un proyecto de origen español, la mayor parte de las investigaciones, se han desarrollado en Europa, aunque recientemente hay publicaciones en Brasil, Colombia y Chile (Braga, 2007; Mello, 2009; Girotto y Mello, 2012; Santos, 2015; Beltrán, Martínez y Torrado, 2015 e Instituto Natura, 2016).

Se podría considerar como antecedente peruano, una investigación realizada por el equipo de la Universidad Marcelino Champagnat en Lima, titulada: "Evaluación de impacto de las Tertulias Literarias Dialógicas en la comprensión lectora”. El estudio se está realizando a lo largo de 2 años y actualmente se encuentra en la mitad del segundo año. La investigación tiene como objetivo medir el impacto de las Tertulias Literarias Dialógicas en la mejora de la comprensión lectora, fluidez lectora y gusto por la lectura en alumnos del Callao. Se desea obtener relaciones de causalidad y atribución de la aplicación de las Tertulias Literarias Dialógicas en la mejora de la comprensión lectora. Es un estudio cuasi experimental con grupo de tratamiento y control. La muestra son alumnos de $2^{\circ}$ de secundaria de 5 escuelas de Callao.

En cuanto antecedentes internacionales, se encuentran estudios cualitativos dentro de la metodología "comunicativa crítica". Esta metodología aparece en el ámbito de las ciencias sociales y aboga por la participación de los sujetos y grupos investigados en todas las fases del proceso investigador (Flecha, 2004). Muchos de ellos son estudios de caso que utilizan instrumentos como entrevistas a profundidad, observación comunicativa, relato comunicativo de vida cotidiana, grupos focales entre otros. 
En el contexto latinoamericano, se han podido encontrar antecedentes principalmente en Brasil. El estudio de Girotto y Mello de 2012 titulado "O ensino da leitura em sala de aula com crianças: a tertúlia literária dialógica”. Este estudio tiene por objetivo ampliar el debate acerca de la lectura de clásicos universales para reforzar aprendizajes y conectarlo con la vida de los estudiantes. Esta tesis doctoral (Universidad Federal de San Carlos) estudia un salón de clases de una escuela en Sao Paulo y utilizó un enfoque de investigación cualitativa llamado comunicativo crítico. Esta investigación realizó un recojo de información del primer semestre del año 2008; aplicó instrumentos como los siguientes: observaciones en clases, los relatos comunicativos de vida y los grupos de discusión comunicativos realizados con las profesoras y con los niños y niñas. Entre sus conclusiones destaca que la Tertulia Literaria Dialógica es un medio eficaz para el empoderamiento de los niños y niñas en el dominio de la lectura y afirma que es un medio útil de aprendizaje de la lectura.

Entre las investigaciones internacionales, encontramos otras en el contexto europeo como la investigación de Flecha, García y Gómez (2013), titulada "Transferencia de Tertulias Literarias Dialógicas a instituciones penitenciarias"; se planteó como objetivo el analizar el material escrito por los participantes sobre la Tertulia Literaria Dialógica -recogido entre 2001 y 2012 - y la correspondencia mantenida desde el centro penitenciario con participantes de otras tertulias. Esta investigación se realizó desde la metodología comunicativo crítica, analizando cinco relatos comunicativos de vida cotidiana de participantes de las tertulias en el centro penitenciario y la de un familiar, así como dos entrevistas en profundidad. Los resultados que describen los investigadores indican un potencial transformador de las tertulias para la reinserción social de los internos, porque enfatizan la dimensión instrumental que acelera el aprendizaje; son creadoras de sentido y establecen lazos de solidaridad entre otros.

Otro estudio de Díez-Palomar, F. J. (2004) titulado "La Enseñanza de las matemáticas en la educación de personas adultas: un modelo dialógico", presentado como tesis doctoral en la Universidad de Barcelona en España, plantea como objetivo analizar algunos procesos afectivos y cognitivos que influyen en el desarrollo de las habilidades comunicativas matemáticas en el proceso de aprendizaje, desde la didáctica de las matemáticas. La investigación se diseñó en la metodología comunicativa crítica, teniendo como muestra los estudiantes de la escuela La Verneda - Sant Marti de España que implementa 
el proyecto de "Comunidades de Aprendizaje". El trabajo se realizó a través de entrevistas, diario de campo, una tertulia comunicativa. En su análisis se consideró, el discurso y el tono del discurso. Entre sus conclusiones presenta que en el aprendizaje dialógico el proceso de enseñanza-aprendizaje debe darse dentro de entornos igualitarios y debe de construirse con "altas expectativas", ya que se cree en la capacidad natural que tienen todas las personas de aprender y en la desaparición de las barreras al aprendizaje a través de la solidaridad, la participación activa e igualitaria de los diferentes actores de la comunidad educativa.

\subsection{El giro dialógico}

En el ámbito escolar, el diálogo, la comunicación y la interacción entre los diferentes actores de la comunidad educativa se vuelven un foco central. Este giro dialógico de las últimas décadas (Flecha, Gómez y Puigvert, 2001) se encuentra con la educación y ha hecho que se empiece a analizar el aprendizaje desde la mirada de las interacciones sociales y la convivencia democrática (Valls, 2000; Gabassa, 2009; Aubert, et al., 2009).

Unas de las primeras referencias sobre este giro dialógico son la teoría de la acción dialógica de Freire (Freire, 1987) donde se resalta la naturaleza dialógica de la persona. Freire teorizó sobre la acción comunicativa con una mirada multicultural, una postura cercana a lo propuesto en el principio de inteligencia cultural en "Comunidades de Aprendizaje". Es importante mencionar también la teoría de la acción comunicativa de Habermas (Habermas, 1987) donde el autor desarrolla una concepción de la competencia comunicativa en la que todas las personas son capaces de lenguaje y de acción. Actualmente, se considera que lo que aprende cada estudiante está menos vinculado con lo que ocurre exclusivamente en el salón de clase y más vinculado con aquello que "ocurre en el aula, el domicilio y la calle." (Castells, Flecha, Freire, Giroux, Macedo y Willis, 1994, citado por Elboj y Oliver, 2003, p. 92).

Ante este nuevo contexto dialógico, la escuela debe cambiar para dar respuesta a las demandas sociales y superar desigualdades educativas que abonan a las desigualdades sociales (Navareño, 2012). La escuela cumple un rol en los procesos de transformación social.

En la actual sociedad del conocimiento, la educación se ha convertido en la clave para la promoción y la inclusión social. Por este motivo, se necesitan nuevos modelos educativos que permitan a todas las personas desarrollar las 
nuevas habilidades y competencias necesarias para enfrentarse a los rápidos cambios sociales que se están produciendo (Elboj y Oliver, 2003, p. 92).

\subsection{Comunidades de Aprendizaje}

Comunidades de Aprendizaje se define como:

Un proyecto de transformación social y cultural de un centro educativo y de su entorno para conseguir una sociedad de la información para todas las personas, basada en el aprendizaje dialógico, mediante una educación participativa de la comunidad, que se concreta en todos sus espacios, incluida el aula. (Valls, 2000, p. 8)

Las Comunidades de Aprendizaje buscan la transformación social y cultural de un centro educativo y de su entorno mediante una educación integrada, participativa y permanente, basada en el aprendizaje dialógico (Elboj, Valls y Fort, 2000) y vinculan a toda la comunidad al proceso educativo en espacios concretos, incluyendo el aula de clases (Ferrada y Flecha, 2008).

1.3.1. Características de las Comunidades de Aprendizaje

Beltrán et al. (2015) indican que todas las escuelas que implementan el proyecto de "Comunidades de Aprendizaje" cumplen cuatro características fundamentales:

- Valoración de la inteligencia cultural de todas las personas adultas (Aubert et al., 2008, Díez-Palomar y Flecha, 2010).

- Transformación del entorno cultural buscando reducir el estado de desigualdad (Duque, Mello y Gabassa, 2009; Gómez, 2011; Santos, 2015).

- Se centran en el aprendizaje dialógico y el cooperativismo (Valls, 2000; Aubert et al., 2008; Gabassa, 2009).

- Promueve la inclusión escolar (Santos, 2015; Gómez et al., 2010; Valls et al., 2014), "eliminando prácticas segregacionistas al considerar que todos pueden aprender y aportar su propio conocimiento y experiencia en un mismo entorno escolar" (Beltrán et al., 2015, p. 59).

En la actualidad el proyecto "Comunidades de Aprendizaje" está siendo 
implementado en España, México, Colombia, Brasil, Chile Argentina y Perú, en más de 970 instituciones educativas en diferentes niveles de implementación (Instituto Natura, 2016).

\subsection{Convivencia democrática}

La convivencia se basa en la práctica de relaciones interpersonales que se modulan y permiten vivir en sociedad. Este proceso se inicia en nuestra vida familiar y se sigue construyendo en la escuela:

Convivir para vivir es un reto en la vida de las personas; nuestras experiencias vitales de socialización se inician en el seno de nuestra familia, conviviendo con nuestros padres, hermanos etc., allí vamos construyendo y desarrollando nuestras primeras habilidades intrapersonales e interpersonales y configurando nuestra actitudes, prejuicios y valores. (Benites, 2011, p. 143)

De manera específica, la publicación conjunta de los Ministerios de Educación y de la Mujer y Poblaciones Vulnerables: Aprendiendo a resolver conflictos en las instituciones educativas: orientaciones para directivos y tutores de primaria y secundaria define la convivencia democrática como:

Conjunto de relaciones interpersonales horizontales, caracterizadas por el respeto y valoración del otro; construida y aprendida en la vivencia cotidiana y el diálogo intercultural en la institución educativa. Favorece el desarrollo de vínculos afectivos e identitarios; así como el desarrollo integral de los y las estudiantes, en un marco de respeto, inclusión y ejercicio de derechos y responsabilidades, contribuyendo a la solución pacífica de conflictos y la construcción de un entorno seguro y protector. (Ramos, Ravello, Rivera, Varillas, Samanez y Morales, 2013, p. 117)

A pesar de que la anterior definición, no está dada dentro del marco teórico del aprendizaje dialógico, consideramos que existen muchas similitudes y que es fácil de incorporar al discurso del proyecto "Comunidades de Aprendizaje", pues existe concordancia con 4 de los 7 principios del aprendizaje dialógico: Diálogo igualitario, igualdad de diferencias, inteligencia cultural y transformación. 


\section{Método}

Esta investigación tiene un alcance descriptivo debido a que busca conocer la relación o grado de asociación que existe entre dos variables en una muestra o contexto en particular, busca describir, analizar e interpretar sistemáticamente un conjunto de hechos o fenómenos en los términos deseados. (Hernández, Fernández y Batista, 2014).

\subsection{Tipo de investigación}

Esta investigación es de tipo básica y sustantiva (Sánchez y Reyes, 2006), así como cuantitativa (Hernández et al., 2014). Se considera básica, puesto que busca describir, explicar y predecir determinadas variables generando aportes al conocimiento teórico de las variables de estudio. Es sustantiva porque busca dar respuesta a problemas teóricos o sustantivos, para describir, explicar, predecir o retrodecir la realidad con lo cual se va en búsqueda de principios y leyes generales que permitan organizar una teoría científica (Sánchez y Reyes, 2006, p.38). También se considera cuantitativa, ya que trabaja con mediciones y estima magnitudes. Las investigaciones cuantitativas buscan identificar diferencias, medir resultados (Hernández et al., 2014).

\subsection{Diseño de investigación}

El diseño de la investigación es no experimental, descriptivo correlacional (Sánchez y Reyes, 2006). Se considera no experimental porque solamente se analizan los datos sin intervenir directamente. Se considera descriptivo; ya que se identifican y describen características del tema de estudio y es correlacional puesto que se establece el grado de variación conjunta que ambas variables de estudio tienen entre sí. El esquema que corresponde a este diseño es el siguiente:

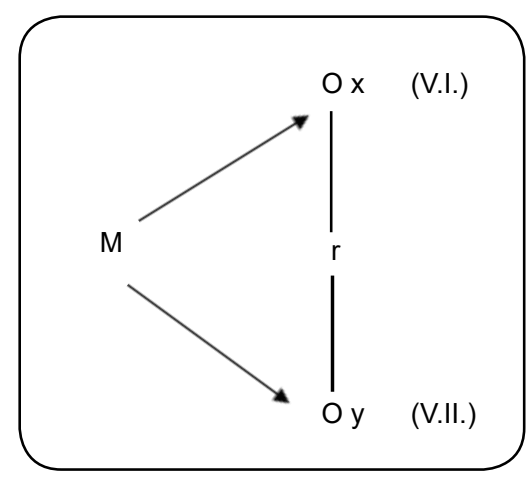


La descripción del esquema en este caso es el siguiente:

$\begin{array}{lll}\text { M } & : & \text { Muestra de investigación } \\ \text { Ox } & : & \text { Variable I Disposición al aprendizaje } \\ \text { Oy } & : & \text { Variable II } \quad \text { Convivencia Democrática } \\ \mathrm{r} & : & \text { Coeficiente de Correlación }\end{array}$

Finalmente, se considera como un estudio de corte transversal porque la recolección de los datos se realizó en un solo momento.

\subsection{Variables}

\section{Variable I: Disposición al aprendizaje}

La disposición al aprendizaje es la "disposición del alumno para estudiar, aprender y desempeñar las acciones propias de un estudiante." (Unidad de Currículum y Evaluación, 2014, p. 16).

Los principios del aprendizaje dialógico vinculados con la disposición al aprendizaje son los siguientes:

- Creación de sentido: "Es un concepto mucho más amplio y del mismo modo que los significados se crean en relación con las otras personas, el sentido también es intersubjetivo" (Aubert et al., 2009, p. 136). La creación de sentido mejora visiblemente la confianza y el empeño de los alumnos en la búsqueda de sus realizaciones personales y colectivas.

- Dimensión Instrumental: se refiere al aprendizaje de aquellos instrumentos fundamentales que son base para poder acceder a los demás aprendizajes y un requisito para obtener una formación de calidad (Elboj, et al. 2006). Aquí tiene principal importancia la lectura como instrumento fundamental para la mayoría de aprendizajes académicos y sociales.

\section{Definición operacional de la variable I}

Para fines de la investigación no se consideraron dimensiones dentro de la variable disposición al aprendizaje. A continuación se desagrega la matriz en la Tabla 1. 
Tabla 1

Operacionalización de la Variable I

\begin{tabular}{llll}
\hline \multicolumn{1}{c}{ Variable I } & \multicolumn{1}{c}{ Dimensiones } & \multicolumn{1}{c}{ Indicadores } & \multicolumn{1}{c}{ Items } \\
\hline $\begin{array}{l}\text { Disposición al } \\
\text { aprendizaje }\end{array}$ & $\begin{array}{l}\text { Disposición al } \\
\text { aprendizaje }\end{array}$ & $\begin{array}{l}\text { Capacidad de } \\
\text { Argumentación }\end{array}$ & 4,8 \\
$\begin{array}{l}\text { Disposición al } \\
\text { aprendizaje }\end{array}$ & $\begin{array}{l}\text { Disposición al } \\
\text { aprendizaje }\end{array}$ & Trabajo en equipo & 5,44 \\
$\begin{array}{l}\text { Disposición al } \\
\text { aprendizaje }\end{array}$ & $\begin{array}{l}\text { Disposición al } \\
\text { aprendizaje }\end{array}$ & Altas expectativas & $12,16,20$ \\
$\begin{array}{l}\text { Disposición al } \\
\text { aprendizaje }\end{array}$ & $\begin{array}{l}\text { Disposición al } \\
\text { aprendizaje }\end{array}$ & Mejora de vocabulario & 24 \\
$\begin{array}{l}\text { Disposición al } \\
\text { aprendizaje }\end{array}$ & $\begin{array}{l}\text { Disposición al } \\
\text { aprendizaje }\end{array}$ & Gusto por la lectura & $28,32,40,54$ \\
$\begin{array}{l}\text { Disposición al } \\
\text { aprendizaje }\end{array}$ & $\begin{array}{l}\text { Disposición al } \\
\text { aprendizaje }\end{array}$ & Extrapolación & 36 \\
$\begin{array}{l}\text { Disposición al } \\
\text { aprendizaje }\end{array}$ & $\begin{array}{l}\text { Disposición al } \\
\text { aprendizaje }\end{array}$ & Interés por la escuela & 52,53 \\
\hline
\end{tabular}

Fuente: elaboración propia

\section{Variable II: Convivencia democrática}

Ramos, Ravello, Rivera, Varillas, Samanez y Morales (2013) definen la convivencia democrática como un conjunto de relaciones interpersonales horizontales, caracterizadas por el respeto y valoración del otro; construida y aprendida en la vivencia cotidiana y el diálogo intercultural en la institución educativa. Favorece el desarrollo de vínculos afectivos e identitarios; así como el desarrollo integral de los y las estudiantes en un marco de respeto, inclusión y ejercicio de derechos y responsabilidades, contribuyendo a la solución pacífica de conflictos y la construcción de un entorno seguro y protector (p. 117).

\section{Definición operacional de la variable II}

Operacionalmente se puede definir la convivencia democrática en las siguientes dimensiones, que responden a los principios del aprendizaje dialógico (ver Tabla 2): 
- Diálogo igualitario: "El diálogo igualitario implica que todas las personas, independientemente de su origen social, étnico, opción ideológica, religión, forma de vida, etc., argumentan y dialogan en un plano de igualdad. Para ello se promociona el valor que tienen los argumentos de la persona que opina y no la posición social" (Duque et al., 2009, p37).

- Transformación: las personas somos seres de transformación y no de adaptación (Freire, 1997). "La educación y el aprendizaje deben ir enfocados hacia el cambio para romper con el discurso de la modernidad tradicional basado en teorías conservadoras que negaban la posibilidad de transformación." (Vila Merino, 2011, p. 12).

- Inteligencia cultural: es más amplia que la inteligencia cognitiva y considera la pluralidad de dimensiones de la interacción humana. Engloba a la inteligencia académica, las capacidades de lenguaje y las acciones propias de las personas, que permiten llegar a acuerdos en diferentes ámbitos sociales (Vila Merino, 2011).

Tabla 2

Operacionalización de la Variable II

\begin{tabular}{llll}
\hline \multicolumn{1}{c}{ Variable II } & \multicolumn{1}{c}{ Dimensiones } & \multicolumn{1}{c}{ Indicadores } & \multicolumn{1}{c}{ Items } \\
\hline Convivencia & Diálogo Igualitario & Escucha con respeto & $1,2,3$, \\
Convivencia & Diálogo Igualitario & Valorar la contribución de todos & 6,14 \\
Convivencia & Diálogo Igualitario & Horizontalidad & 7,9 \\
Convivencia & Transformación & $\begin{array}{l}\text { Valoración de lo propio/local } \\
\text { (identidad) }\end{array}$ & 10,17 \\
Convivencia & Diálogo Igualitario & Miedo a hablar & $11,13,33$ \\
Convivencia & Transformación & Bullying /Violencia & $15,31,34$ \\
Convivencia & Transformación & Interacciones con la comunidad & 18 \\
Convivencia & Transformación & Interacciones docente-estudiante & $19,21,22$ \\
Convivencia & Transformación & Interacciones estudiante- & $23,25,26,27$ \\
Convivencia & Transformación & Ganas de cambio & 29,30 \\
Convivencia & Transformación & Liderazgo dialógico & $35,37,55$
\end{tabular}




\begin{tabular}{llll} 
Convivencia & Transformación & $\begin{array}{l}\text { Participación en la toma de } \\
\text { decisiones }\end{array}$ & $38,39,41$ \\
Convivencia & Transformación & Ayuda al otro & $42,45,47$ \\
Convivencia & Transformación & Voluntariado & 43,36 \\
Convivencia & Transformación & Valoración de las diferencias & $48,59,51$ \\
Convivencia & Transformación & Respeto & 49 \\
\hline
\end{tabular}

Fuente: elaboración propia

\subsection{Población, muestra y muestreo}

\section{Población}

La población de estudio está conformada por los estudiantes de las instituciones educativas públicas que participan en el Proyecto "Comunidades de Aprendizaje" en la modalidad de transformación y que implementan las Tertulias Literarias Dialógicas. En total son 43 instituciones educativas en las regiones de Lima, Callao, Piura, Cusco, Apurímac, Huancavelica y Puno. Todas las instituciones educativas brindan educación básica regular, ya sea en ámbitos urbanos o rurales.

\section{Muestra}

El estudio es censal; la muestra es igual a la población participante y está conformada por todos los estudiantes de $3^{\circ}$ de primaria a $5^{\circ}$ de secundaria que aplican las Tertulias Literarias Dialógicas en alguna de las 43 escuelas del proyecto. De este total, 20 se encuentran en zonas rurales y 23 en zonas urbanas.

Del total de las escuelas, 7 son solo escuelas primarias, 22 son solamente secundarias y 14 tienen ambos niveles. Si se considera el año de implementación del proyecto, 19 se encuentran en su primer año de implementación y 24 en su segundo año. Se encuentran distribuidas en siete regiones (Tabla 3), tomando en cuenta ambos sexos (Tabla 4) y niveles educativos (Tabla 5). 
Tabla 3

Caracterización general de las escuelas

\begin{tabular}{ccccc}
\hline Escuela & Región & Área & Nivel & Total estudiantes encuestados \\
\hline 1. & Apurímac & Rural & Secundaria & 91 \\
2. & Callao & Urbana & Primaria y Secundaria & 1683 \\
3. & Cuzco & Rural & Secundaria & 1248 \\
4. & Lima & Urbana & Primaria y Secundaria & 3605 \\
5. & Sullana & Urbana & Primaria y Secundaria & 2621 \\
6. & Puno & Rural & Secundaria & 108 \\
7. & Huancavelica & Rural & Secundario & 381 \\
& & & Total & 9944 \\
\hline
\end{tabular}

Fuente: elaboración propia

Tabla 4

Población de estudiantes por región y sexo

\begin{tabular}{lccc}
\hline \multicolumn{1}{c}{ Región } & Mujer & Hombre & Total \\
\hline Apurímac & 70 & 85 & 155 \\
Callao & 872 & 861 & 1733 \\
Cusco & 592 & 656 & 1248 \\
Huancavelica & 181 & 205 & 386 \\
Lima & 1826 & 1811 & 3637 \\
Piura & 1259 & 1418 & 2677 \\
Puno & 31 & 77 & 108 \\
Total & 4831 & 5113 & 9944 \\
\hline
\end{tabular}

Fuente: elaboración propia

Tabla 5

Población de estudiantes por región y nivel educativo

\begin{tabular}{lccc}
\hline Región & Primaria & Secundaria & Total \\
\hline Apurímac & 0 & 155 & 155 \\
Callao & 1076 & 657 & 1733 \\
Cusco & 0 & 1248 & 1248 \\
Huancavelica & 0 & 386 & 386 \\
Lima & 1937 & 1700 & 3637 \\
Piura & 1177 & 1500 & 2677 \\
Puno & 0 & 108 & 108 \\
Total estudiantes & 4190 & 5754 & 9944 \\
\hline
\end{tabular}

Fuente: Elaboración propia 
El criterio de inclusión en el recojo de información fue la participación del proceso de Transformación en "Comunidades de Aprendizaje", tanto del primer año de implementación como del segundo año y que realicen las Tertulias Literarias. Dado que se requiere un nivel de comprensión lectora para poder resolver la encuesta, no participan los alumnos de inicial, ni de $1^{\circ}$ y $2^{\circ}$ grado de primaria.

\subsection{Instrumentos}

Se elaboró una encuesta tipo Likert con algunas preguntas abiertas. Los ítems de la encuesta fueron construidos considerando la operacionalización de las variables I y II, son en total 53 ítems (39 ítems sobre convivencia y 14 ítems sobre disposición al aprendizaje); los cuales presentan como alternativas de respuesta una escala cerrada de frecuencia (nunca, algunas veces, muchas veces, siempre)

La confiabilidad del instrumento se calculó mediante los coeficientes de consistencia interna de las escalas Alfa de Cronbach $(\alpha)$. Este coeficiente fue desarrollado por J. L Cronbach y requiere una sola administración del instrumento, generando valores entre 0 y 1 . La fórmula para calcular este coeficiente es:

$$
\alpha=\frac{K}{K-1}\left[1-\frac{\sum S_{i}^{2}}{S_{t}^{2}}\right]
$$

Donde:

$\mathrm{K} \quad$ : Es el número de ítems

$\sum \mathrm{S}_{\mathrm{i}}{ }^{2}$ : Sumatoria de varianzas de los ítems

$\mathrm{S}_{\mathrm{t}}^{2} \quad$ : Varianza de la suma de los ítems

$\alpha \quad$ : Coeficiente de Alfa de Cronbach

Al reemplazar los valores para cada de una las variables, se obtuvieron los resultados que se pueden apreciar en la Tabla 9. 
Tabla 9

Resultados de la confiabilidad en la aplicación del piloto

\begin{tabular}{lcc}
\hline \multicolumn{1}{c}{ Variables } & Confiabilidad & N de elementos \\
\hline Convivencia & 0,793 & 39 \\
Disposición al aprendizaje & 0,614 & 14 \\
\hline
\end{tabular}

Fuente: elaboración propia

No hay un único criterio sobre puntos referenciales para la confiabilidad. Es importante mencionar que Huh, Delorme y Reid (2006) indican que el valor de fiabilidad en una investigación exploratoria debe ser igual o mayor a 0,6 (como es en la presente investigación) y que en estudios confirmatorios debe estar entre 0,7 y 0,8 De igual forma, Nunnally (1967) indica que un valor entre 0.5 y 0.6 puede ser suficiente en las primeras fases de investigaciones. Algunos autores como Hernández et al. (2006) consideran que hay confiabilidad desde valores de 0,60 (ver Tabla 10). Considerando a estos autores, el instrumento de evaluación de la convivencia tienen un nivel de confiabilidad excelente, mientras que el instrumento de evaluación de la disposición al aprendizaje tienen un nivel confiable (ver Tabla 10).

Tabla 10

Valores de los niveles de confiabilidad

\begin{tabular}{cc}
\hline Valores & Nivel de confiabilidad \\
\hline 0,53 a menos & Confiabilidad nula \\
0,54 a 0,59 & Confiabilidad baja \\
0,60 a 0,65 & Confiable \\
0,66 a 0,71 & Muy confiable \\
0,72 a 0,99 & Excelente confiabilidad \\
1 & Confiabilidad perfecta \\
\hline
\end{tabular}

Fuente: Adaptado de Hernández et al. (2006)

También se analizó la correlación ítem-test y se identificaron las preguntas que tenían una correlación menor a 0,2 ; por este motivo se tuvieron que retirar siete ítems en el instrumento de evaluación de la convivencia democrática 
(ítems $\mathrm{N}^{\circ} 6,7,9,11,14$ y 33) y un ítem en el instrumento de evaluación de la disposición al aprendizaje (ítem $\mathrm{N}^{\circ} 4$ ); para mayor detalle sobre los mismos ver Tabla 1. Con estas consideraciones y modificaciones se preparó una versión final de la encuesta con el equipo central del proyecto "Comunidades de Aprendizaje" de Perú.

Al aplicarse la prueba a toda la muestra se observó una mejora considerable en los resultados de confiabilidad como puede verse en la Tabla 11.

Tabla 11

Resultados de confiabilidad en la aplicación de los instrumentos

\begin{tabular}{lcc}
\hline \multicolumn{1}{c}{ Variables } & Alfa de Cronbach & N de elementos \\
\hline Convivencia & 0.83 & 33 \\
Disposición al aprendizaje & 0,69 & 13 \\
\hline
\end{tabular}

Fuente: elaboración propia

\section{Resultados}

Los resultados obtenidos de la aplicación de los instrumentos se analizaron primero de forma descriptiva y luego en el nivel inferencial en función de las hipótesis y objetivos propuestos. A nivel descriptivo, se han utilizado frecuencias y porcentajes para determinar los niveles predominantes de las variables convivencia democrática y disposición al aprendizaje en los estudiantes de tercer grado de primaria a quinto grado de secundaria en la red de escuelas que participan del proyecto "Comunidades de Aprendizaje" en el Perú. En el nivel inferencial, se utilizó estadística no paramétrica para establecer la correlación entre las variables (coeficiente de correlación rho de Spearman) y para determinar diferencias significativas entre muestras independientes (prueba U de Mann-Whitney).

\subsection{Estadísticas y análisis de datos a nivel descriptivo}

Luego de la aplicación se procedió al procesamiento de las encuestas; en un primer momento se revisaron las 9944 encuestas aplicadas y se encontró que el 3,48\% (346 encuestas) se encontraban incompletas, motivo por el cual se tuvieron que retirar, quedando 9598 encuestas $(96,52 \%)$ para ser procesadas estadísticamente. 
2.1.1. Resultados de la variable convivencia democrática

Se analizó la suma total de los valores de la variable convivencia democrática. El valor mínimo posible es de 33 y el máximo posible es de 132. Los valores de los descriptivos estadísticos generales se muestran en la Tabla 12.

Tabla 12

Resultados descriptivos generales de las variables

\begin{tabular}{lcc}
\hline Descriptivos & Suma total convivencia & Suma total disposición al aprendizaje \\
\hline Media & 99,0924 & 41,1044 \\
Mediana & 99 & 41 \\
Varianza & 152,39 & 31,13 \\
Desviación & 12,34 & 5,58 \\
Mínimo & 42 & 14 \\
Máximo & 132 & 52 \\
Rango & 90 & 38 \\
\hline
\end{tabular}

Fuente: elaboración propia

De igual forma se analizaron los resultados en función de cuatro niveles de convivencia democrática determinados en función de la suma total de la variable. Las frecuencias para el nivel bajo fueron de 12 estudiantes; para el nivel medio, de 992 estudiantes; para el nivel alto, 6438 estudiantes; y para el nivel muy alto, 2156 estudiantes. En la Figura 1, se puede ver la distribución de las frecuencias de los niveles y sus porcentajes. Es importante notar que $89.54 \%$ de los estudiantes se encuentran en niveles altos o muy altos 


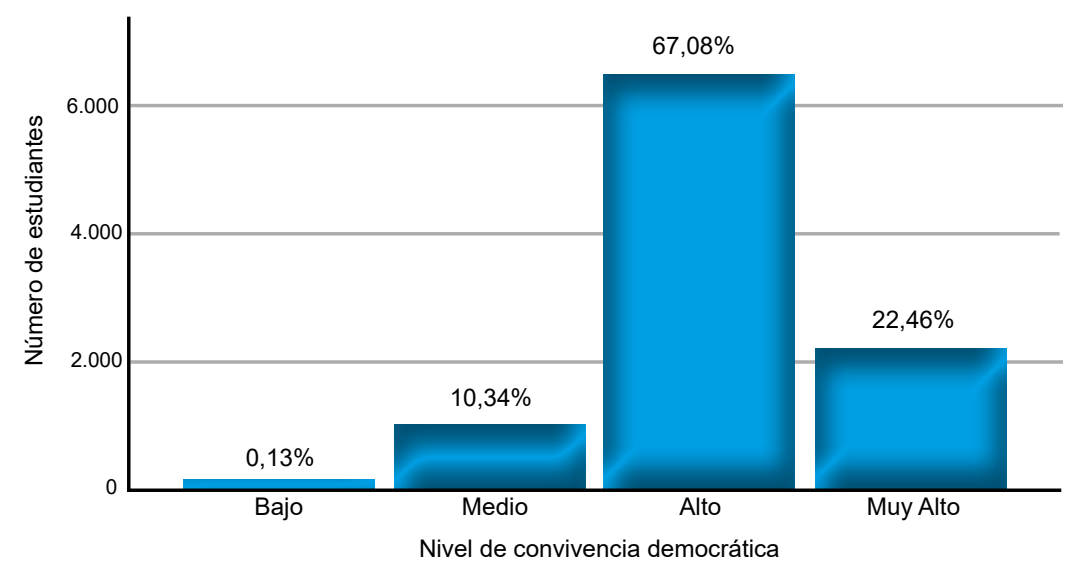

Figura 1. Nivel de convivencia democrática considerando la suma total de la variable.

\subsubsection{Resultados de la variable disposición al aprendizaje}

Se analizó la suma total de los valores de la variable disposición al aprendizaje. El valor mínimo posible es 13 y el máximo posible es 52. Los valores de los descriptivos estadísticos generales se muestran en la Tabla 12. De igual forma, se analizaron los resultados en función de cuatro niveles de disposición al aprendizaje; estos determinados en función de la suma total de la variable. Las frecuencias para el nivel bajo fueron de 18 estudiantes; para el nivel medio, 662 estudiantes; para el nivel alto,4827 estudiantes; y para el nivel muy alto, 4091 estudiantes. En la figura 2, se puede ver la distribución de las frecuencias de los niveles y sus porcentajes. Es importante notar que el 92,9\% de los estudiantes se encuentran en el nivel alto o muy alto.

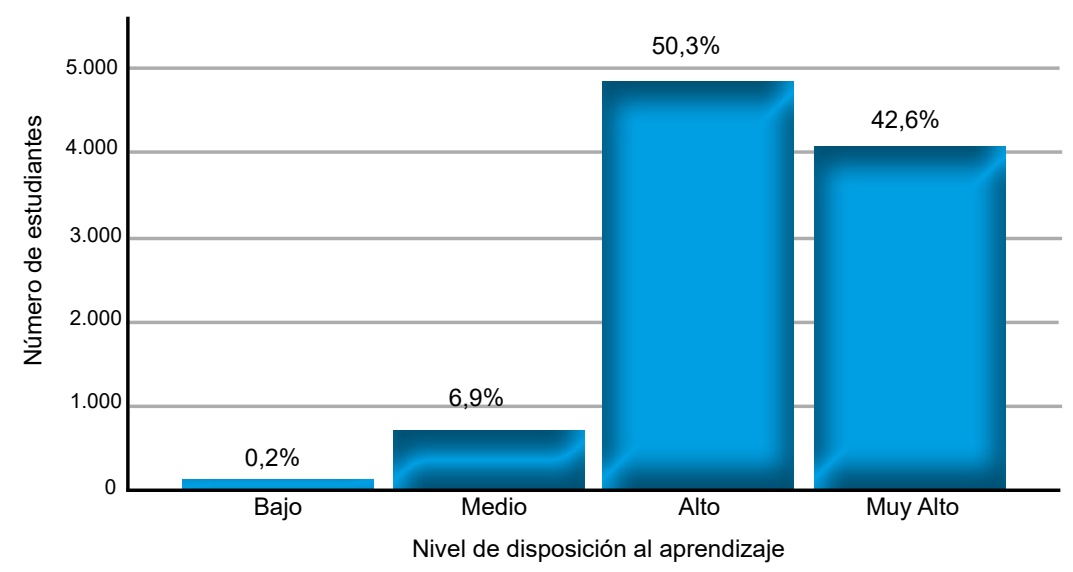

Figura 2. Nivel de convivencia disposición al aprendizaje considerando la suma total de la variable. 


\subsection{Estadísticas y análisis de datos a nivel inferencial}

Para realizar el análisis inferencial fue necesario determinar el tipo de distribución de los resultados de ambos instrumentos. Esto se realizó mediante la prueba Kolmogorov - Smirnov debido al tamaño de la muestra (más de 50 datos). Esta prueba permite medir la concordancia entre la distribución de un conjunto de datos y una distribución teórica específica (García, González y Jornet, 2010). En la Tabla 13 se observa el valor de significancia del estadístico de prueba de normalidad; el que para ambas encuestas, es de 0,00. Esto demuestra que los resultados no tienen distribución normal, motivo por el cual se deben de utilizar pruebas estadísticas no paramétricas para realizar el análisis inferencial como son el coeficiente de correlación rho de Spearman y la prueba U de Mann-Whitney.

Tabla 13

Resumen de prueba de distribución de las variables

\begin{tabular}{lccc}
\hline \multicolumn{1}{c}{ Hipótesis nula } & Prueba & Sig. & Decisión \\
\hline $\begin{array}{l}\text { Distribución de la variable convivencia es } \\
\text { normal con la media 0.751 y la desviación } \\
\text { estándar 0.09. }\end{array}$ & $\begin{array}{c}\text { Prueba de Kolmogorov- } \\
\text { Smirnov para una muestra }\end{array}$ &, $000^{*}$ & $\begin{array}{c}\text { Rechazar la } \\
\text { hipótesis nula. }\end{array}$ \\
$\begin{array}{l}\text { Distribución de la variable disposición al } \\
\text { aprendizaje es normal con la media 0.790 }\end{array}$ & $\begin{array}{c}\text { Prueba de Kolmogorov- } \\
\text { Smirnov para una muestra }\end{array}$ &, $000^{*}$ & $\begin{array}{c}\text { Rechazar la } \\
\text { hipótesis nula. }\end{array}$ \\
\hline
\end{tabular}

* Lilliefors corregida

Fuente: elaboración propia

\subsubsection{Contrastación de la hipótesis general}

Para contrastar la hipótesis general se utilizó el coeficiente rho de Spearman sobre los resultados de las encuestas de las dos variables; aquí se encontró que tienen una correlación de 0,678 a un nivel de significatividad del 0,00 (ver Tabla 14). Si consideramos los niveles establecidos (por Mondragón, 2014, p.100 y Fernández et al., 2014, p. 305), se puede decir que el valor de la correlación es positivo y considerable, comprobándose la hipótesis general de trabajo. 
Tabla 14

Correlación entre las variables

\begin{tabular}{|c|c|c|c|}
\hline Puntajes & Elementos & $\begin{array}{l}\text { Puntaje de la variable } \\
\text { convivencia }\end{array}$ & $\begin{array}{l}\text { Puntaje de la dimensión } \\
\text { Disposición al aprendizaje }\end{array}$ \\
\hline \multirow{3}{*}{$\begin{array}{l}\text { Puntaje de } \\
\text { la variable } \\
\text { convivencia }\end{array}$} & $\begin{array}{l}\text { Coeficiente de } \\
\text { correlación }\end{array}$ & 1 &, $678 * *$ \\
\hline & Sig. (bilateral) & . & 0 \\
\hline & $\mathrm{N}$ & 9598 & 9598 \\
\hline
\end{tabular}

Fuente: elaboración propia

\title{
2.3. Contrastación de las hipótesis específicas
}

Las hipótesis específicas se han construido en torno a la comparación de muestras independientes para cada una de las variables y para poder contrastarlas se utilizó la prueba U de Mann-Whitney.

\subsubsection{Disposición al aprendizaje en hombres y mujeres}

\begin{abstract}
Al comparar los resultados obtenidos para la variable disposición al aprendizaje y el sexo de los estudiantes, se encuentra que muestran diferencias estadísticamente significativas $(\boldsymbol{\rho}=0)$. Se observa además que el rango promedio de las mujeres es mayor que el de los hombres. La distribución de los puntajes obtenidos se muestra en la Tabla 15.
\end{abstract}

Tabla 15

Disposición al aprendizaje vs. sexo

\begin{tabular}{lcccc}
\hline & Sexo & N & Rango promedio & Suma de rangos \\
\hline Puntaje de la variable & Mujer & 4681 & 4982,15 & 23321447,5 \\
disposición al & Hombre & 4917 & 4625,62 & 22744153,5 \\
aprendizaje & Total & 9598 & & \\
\hline
\end{tabular}

Por estos motivos se acepta la hipótesis alternativa con significancia menor a 0.05 .

Fuente: elaboración propia 


\section{Convivencia democrática en hombres y mujeres}

Al comparar los resultados obtenidos para la variable convivencia democrática y el sexo de los estudiantes, se evidencian diferencias estadísticamente significativas $(\boldsymbol{\rho}=0)$. Se observa, además, que el rango promedio de las mujeres es mayor que el de los hombres. La distribución de los puntajes obtenidos se muestra en la Tabla 16.

Tabla 16

Convivencia vs. sexo

\begin{tabular}{|c|c|c|c|c|c|}
\hline & Sexo & $\mathrm{N}$ & \multicolumn{2}{|c|}{ Rango promedio } & Suma de rangos \\
\hline \multirow{3}{*}{$\begin{array}{l}\text { Puntaje de } \\
\text { la variable } \\
\text { convivencia }\end{array}$} & Mujer & & 4681 & 4965,72 & 23244526,5 \\
\hline & Hombre & & 4917 & 4641,26 & 22821074,5 \\
\hline & Total & & 9598 & & \\
\hline
\end{tabular}

Fuente: elaboración propia

Por estos motivos se acepta la hipótesis alternativa, ya que se presenta una significancia menor a 0.05 .

\section{Disposición al aprendizaje en estudiantes de primaria y secundaria}

Al comparar los resultados obtenidos para la variable disposición al aprendizaje y el nivel educativo en el que estudian los alumnos, se evidencian diferencias estadísticamente significativas $(\boldsymbol{\rho}=0)$. Se observa además que el rango promedio de los alumnos de primaria es mayor que el mismo valor para secundaria. La distribución de los puntajes obtenidos se muestra en la Tabla 17.

Tabla 17

Disposición al aprendizaje vs. nivel educativo

\begin{tabular}{lcccc}
\hline & Nivel educativo & N & Rango promedio & Suma de rangos \\
\hline Puntaje de la variable & Primaria & 3934 & 5298,68 & 20845014 \\
convivencia & Secundaria & 5664 & 4452,79 & 25220587 \\
& Total & 9598 & & \\
\hline
\end{tabular}

Fuente: elaboración propia 
Por estos motivos se acepta la hipótesis alternativa, puesto que se presenta una significancia menor a 0.05 .

\section{Convivencia democrática en estudiantes de primaria y secundaria}

Al comparar los resultados obtenidos para la variable convivencia democrática y el nivel educativo en el que estudian los alumnos se evidencian diferencias estadísticamente significativas $(\boldsymbol{\rho}=0)$. Se observa, además, que el rango promedio de los alumnos de primaria es mayor que el mismo valor para secundaria. La distribución de los puntajes obtenidos se muestra en la Tabla 18.

Tabla 18

Convivencia democrática vs. nivel educativo

\begin{tabular}{llccc}
\hline & \multicolumn{1}{c}{ Tipo de zona } & N & Rango promedio & Suma de rangos \\
\hline Puntaje de la variable & Primaria & 3934 & 4970,48 & 19553855,5 \\
convivencia & Secundaria & 5664 & 4680,75 & 26511745,5 \\
& Total & 9598 & 9598 & \\
\hline
\end{tabular}

Fuente. elaboración propia

Por estos motivos se acepta la hipótesis alternativa, pues, se presenta una significancia menor a 0.05 .

\section{Disposición al aprendizaje entre estudiantes de zonas urbanas y rurales}

Al comparar los resultados obtenidos para la variable disposición al aprendizaje entre estudiantes de zonas urbanas y rurales se evidencian diferencias estadísticamente significativas $(\boldsymbol{\rho}=0)$. Se observa, además, que el rango promedio de los alumnos de zonas rurales es mayor que el mismo valor para alumnos de zonas urbanas. La distribución de los puntajes obtenidos se muestra en la Tabla 19. 
Tabla 19

Disposición al aprendizaje entre estudiantes de zonas urbanas y rurales

\begin{tabular}{|c|c|c|c|c|c|}
\hline & Tipo de zona & $\mathrm{N}$ & Rang & medio & Suma de rangos \\
\hline \multirow{3}{*}{$\begin{array}{l}\text { Puntaje de la variable } \\
\text { disposición al } \\
\text { aprendizaje }\end{array}$} & Urbano & & 7742 & 4726,59 & 36593254,5 \\
\hline & Rural & & 1856 & 5103,63 & 9472346,5 \\
\hline & Total & & 9598 & & \\
\hline
\end{tabular}

Fuente: elaboración propia

Por estos motivos, se acepta la hipótesis alternativa, ya que, se presenta una significancia menor a 0.05 .

\section{Convivencia democrática entre estudiantes de zonas urbanas y rurales}

Al comparar los resultados obtenidos para la variable convivencia democrática entre estudiantes de zonas urbanas y rurales, se evidencian diferencias estadísticamente significativas $(\boldsymbol{\rho}=0,01)$. Se observa, además, que el rango promedio de los alumnos de zonas rurales es mayor que el mismo valor para alumnos de zonas urbanas. La distribución de los puntajes obtenidos se muestra en la Tabla 20.

Tabla 20

Convivencia democrática entre estudiantes de zonas urbanas y rurales

\begin{tabular}{|c|c|c|c|c|c|}
\hline & Tipo de zona & $\mathrm{N}$ & Rang & medio & Suma de rangos \\
\hline \multirow{3}{*}{$\begin{array}{l}\text { Puntaje de la variable } \\
\text { convivencia }\end{array}$} & Urbano & & 7742 & 4752,26 & 36791988,5 \\
\hline & Rural & & 1856 & 4996,56 & 9273612,5 \\
\hline & Total & & 9598 & & \\
\hline
\end{tabular}

Fuente: elaboración propia

Por estos motivos se acepta la hipótesis alternativa; puesto que se presenta una significancia menor a 0.05 . 


\section{Discusión y Conclusiones}

Después del análisis realizado, podemos observar que existe una relación entre la convivencia y la disposición al aprendizaje, aceptando la hipótesis de trabajo. Asimismo, se afirma que existe una relación positiva considerable y altamente significativa entre la convivencia y la disposición al aprendizaje en los estudiantes de $3^{\circ}$ de Primaria a $5^{\circ}$ de Secundaria de la red de escuelas que participan del proyecto.

Además, se observa que existe una diferencia significativa en la disposición al aprendizaje entre estudiantes hombres y mujeres de la red de escuelas que participan en el proyecto. En consecuencia, se rechaza la hipótesis nula y se acepta la hipótesis de trabajo; la disposición al aprendizaje en las estudiantes mujeres es mayor que la de los estudiantes varones.

Existe una diferencia significativa en la disposición al aprendizaje y en la convivencia democrática entre estudiantes de primaria y secundaria de la red de escuelas. Existe una diferencia significativa en la convivencia democrática entre estudiantes de primaria y secundaria de la red de escuelas que participan en el proyecto "Comunidades de Aprendizaje".

Existe una diferencia significativa en la disposición al aprendizaje entre estudiantes de zonas urbanas y rurales de la red de escuelas que participan en el proyecto "Comunidades de Aprendizaje". En consecuencia, se rechaza la hipótesis nula y se acepta la hipótesis de trabajo; la disposición al aprendizaje en los estudiantes de zonas rurales es mayor que la de los estudiantes de zonas urbanas.

Se recomienda complementar el presente estudio con una investigación cualitativa para generar una mayor comprensión de las percepciones de los participantes y de los procesos que se presentan en la escuela. Revisar el instrumento nuevamente con el equipo central del proyecto "Comunidades de Aprendizaje". Asimismo, se pueden generar más ítems para evaluar la variable disposición al aprendizaje, el instrumento debería llegar a tener de manera ideal un alfa de Cronbach de 0.8.

Establecer un esquema de evaluación dentro del proyecto "Comunidades de Aprendizaje" y continuar con la aplicación del instrumento en las nuevas escuelas que se incorporan a la red de "Comunidades de Aprendizaje", de 
manera muestral, dado el alto costo de realizarlo de forma censal. En el presente trabajo se estudia la relación entre convivencia y disposición al aprendizaje; se recomienda explorar la vinculación de los resultados de convivencia con los resultados de las pruebas censales nacionales. Así mismo, sería interesante realizar un análisis más profundo y detallado por regiones y escuelas, vinculando los resultados con factores asociados.

\section{Referencias}

Aldana, C. (2006). Aprender a convivir en un mundo de violencia. Cuadernos de pedagogía, 359, $28-31$.

Arancibia, M. (2014). Diagnóstico e intervención en escuelas desde la política de Convivencia Escolar y enfoque de Derechos Humanos. Revista Electrónica Actualidades Investigativas en Educación, 14(3), 1-18. Recuperado de: http://www.redalyc.org/pdf/447/44732048005.pdf

Aubert, A., Flecha A., García, C., Flecha, R. y Racionero, S. (2008). Aprendizaje dialógico en la sociedad de la información. Barcelona: Hipatia.

Aubert, A., García, C. y Racionero, S. (2009). El aprendizaje dialógico. Cultura y Educación. 21(2), 129-139. Recuperado de: http://personal. us.es/aguijim/05_06_Aprendizaje_dialogico.pdf

Barletti, J. (2009). La alternancia en la educación secundaria rural. Tarea, 72, 64-68. Recuperado de: http://tarea.org.pe/images/Tarea72_Jose_ Barletti.pdf

Beck, U.y Beck-Gernsheim, E. (2003).La individualización: el individualismo institucionalizado y sus consecuencias sociales y políticas. Barcelona: Ediciones Paidós Ibérica.

Beltrán, Y., Martínez, Y. y Torrado, O. (2015). Creación de una comunidad de aprendizaje: una experiencia de educación inclusiva en Colombia. Revista Encuentros, 13(2), 57-72. Recuperado de: http://dx.doi. org/10.15665/re.v13i2.498 
Benites, L. (2011). Convivencia escolar y calidad educativa. Cultura (25): pp. 143-164.

BBC (23 de Febrero de 2017). Estos son los 10 países del mundo con más inmigrantes. BBC Mundo. Recuperado de: http://www.bbc.com/ mundo/noticias-internacional-39059460

Braga, F.M. (2007). Comunidades de aprendizagem : uma única experiência em dois países (Brasil e Espanha) em favor da participação da comunidade na escola e da melhoria da qualidade do ensino. (Tesis Doctoral), Universidade Federal de São Carlos, São Carlos. Recuperado de: http://comunidadesdeaprendizaje.net/wp-content/uploads/2012/04/ tese-final-fabiana.pdf

Carretero, A. (Coord.) (2008). Vivir Convivir: convivencia intercultural en centros de educación primaria. Granada: Andalucía Acoge.

Casamitjana, M., Puigvert, L., Soler, M. y Tortajada, I. (2000). Investigar y transformar: CREA Centro de Investigación Social y Educativa. Cultura y Educación, 17(18), 117-129.

Castells, M. (2004). La era de la información: economía, sociedad y cultura. Vol. 3. México: Siglo XXI.

Castells, M., Flecha, R., Freire, P., Giroiux, H., Macedo, D. y Willis, P. (1994). Nuevas perspectivas críticas en educación. Barcelona: Paidós.

Colombo, G. (2011). Violencia Escolar y Convivencia Escolar: Descubriendo estrategias en la vida cotidiana escolar. Revista Argentina de Sociología, 8-9(15-16), 81-104.

De la Fuente, J., Peralta, F.J. y Sánchez, M.D. (2006). Valores sociopersonales y problemas de convivencia en la Educación Secundaria. Revista Electrónica de investigación Psicoeducativa, 9, 171-200. Recuperado de: http://www.investigacion-psicopedagogica.org/revista/articulos/9/ espannol/Art_9_118.pdf

Díaz, S. \& Sime, L. (2016). Convivencia escolar: una revisión de estudios de la educación básica en Latinoamérica. Revista Virtual Universidad Católica del Norte, 49, 125-145.. Recuperado de http://revistavirtual. ucn.edu.co/index.php/RevistaUCN/article/view/801/1321 
Diez-Palomar, F. (2004). La Enseñanza de las matemáticas en la educación de personas adultas: un modelo dialógico. (Tesis doctoral) Universitat de Barcelona, Barcelona.

Díez-Palomar, J. y Flecha García, R. (2010). Comunidades de Aprendizaje: un proyecto de transformación social y educativa. Revista Interuniversitaria de Formación del Profesorado, 24 (1), 19-30.

Duque, E., Mello, R. y Gabassa, V. (2009). Aprendizaje dialógico. Base teórica de las comunidades de aprendizaje. Aula de Innovación Educativa, $187,36-41$.

Elboj, C. y Oliver, E. (2003). Las comunidades de aprendizaje: Un modelo de educación dialógica en la sociedad del conocimiento. Revista Interuniversitaria de Formación del Profesorado, 17(3), 91-103.

Elboj, C., Puigdellivol, I, Soler, M. y Valls, R. (2006). Las comunidades de aprendizaje: Transformar la educación. Barcelona: Graó.

Elboj, C., Valls, R. y Fort, M. (2000). Comunidades de aprendizaje. Una práctica educativa para la sociedad de la información. Cultura y Educación, 12(1-2), 129-141.

Fairstein, G. y Gyssels, S. (2003): ¿Cómo se aprende? Colección Programa Internacional de Formación de Educadores Populares, Formación Pedagógica. Federación Internacional de Fe y Alegría.

Ferrada, D. y Flecha, R. (2008). El modelo dialógico de la pedagogía: un aporte desde las experiencias de comunidades de aprendizaje. Estudios Pedagógicos, 34(1), 41-61.

Fernández R., Garvin R. y González, V. (2012). Tertulias pedagógicas dialógicas: Con el libro en la mano. Revista electrónica interuniversitaria de formación del profesorado, 15(4), 113-118. Recuperado de: http:// www.aufop.com/aufop/uploaded_files/articulos/1364431359.pdf

Ferreyra, A. (2016). La transformación soñada: De un centro rural agrupado a una comunidad de aprendizaje. (Tesis doctoral). Universidad de Valladolid, Valladolid. Recuperado de: https:/uvadoc.uva.es/ bitstream/10324/16496/1/Tesis884-160309.pdf 
Fierro, C., Carbajal, P y Martínez-Parente, R. (2010). Ojos que sí ven. Casos para reflexionar sobre la convivencia en la escuela. México: SM, $3^{\mathrm{a}}$. Reimpresión.

Fierro, C. y Fortoul, B. (2013). Convivencia escolar y aprendizaje. Revista Latinoamericana de Educación Inclusiva, 6 (2), 17-22.

Fierro, C y Tapia, G. (2012). Indicadores de convivencia. Concepto operacional, subdimensiones y categorías. Reporte técnico. México: UIA León-CONCYTEG.

Flecha, R. (2004). ¿Por qué Paulo Freire es el principal pedagogo de la actual sociedad de la información? En A. M. Araújo Freire, I. A. de Oliveira, \& R. Luiz Machado (Eds.). La Pedagogía de la liberación en Paulo Freire Crítica y fundamentos Vol. 3, 171-174). Barcelona: Graó.

Flecha, R., García, R. y Gómez, A. (2013). Transferencia de tertulias literarias dialógicas a instituciones penitenciarias. Revista de Educación, 360, 140-161. Recuperado de: http:/www.mecd. gob.es/dctm/revista-de-educacion/articulosre360/re36008. pdf?documentId=0901e 72b814a77e8

Flecha, R., Gómez, J. y Puigvert, L.(2001). Teoría sociológica contemporánea. Barcelona: Paidós.

Flecha, R., Vargas J. y Dávila, A. (2004). Metodología comunicativa crítica en la investigación en ciencias sociales: la investigación workalo. Revista de relaciones laborales, 11, 21-33.

Folgueiras, P. (2011). Las comunidades de aprendizaje. La escuela de adultos de la Verneda. Una experiencia de comunidades de aprendizaje. Tendencias Pedagógicas, 18, 251-267.

Freire, P. (1985). Pedagogía del oprimido. Montevideo: Siglo XXI Editores. Recuperado de: http://www.servicioskoinonia.org/biblioteca/general/ FreirePedagogiadelOprimido.pdf

Freire, P. (1997). Pedagogía de la autonomía. Madrid: Siglo XXI Editores.

Gabassa, V. (2009). Comunidades de aprendizagem: a construção da dialogicidade na sala de aula. (Tesis Doctoral). Universidade Federal de São Carlos: São Carlos. 
García, R., González, J. y Jornet, J. (2010). SPSS: Pruebas no paramétricas: Kolmogorov Smirnov. Grupo de Innovación Educativa, Innova mide. Recuperado el 1 de Junio de 2015, de http://www. uv.es/innomide/spss/ SPSS/SPSS_0802A.pdf

Gardner, H. (2003). La inteligencia reformulada. Las inteligencias múltiples en el siglo XXI. Barcelona: Paidós [V.O. Intelligence Reframed: Multiple Intelligences for the 21st Century. Nueva York: Basic Books, 1999].

Giddens, A. (1995). Modernidad e identidad del yo. Barcelona: Península.

Girotto, V.C. y Mello, R.R. (2012). O ensino da leitura em sala de aula com crianças: a tertúlia literária dialógica. Inter-Ação, 37 (1), 67-84.

Gómez, A., Mello, R., Santa Cruz, I. y Sordé, T. (2010). De las experiencias de Comunidades de Aprendizaje a las políticas basadas en sus éxitos. Revista Interuniversitaria de Formación del Profesorado, 67 (24,1), 113-126 Recuperado de: https:/dialnet.unirioja.es/descarga/ articulo/3148968.pdf

Habermas, J. (1987). Teoría de la Acción Comunicativa. Vol. I: Racionalidad de la acción y racionalización social. Vol. II: Crítica de la razón funcionalista. Madrid: Taurus.

Hernandez, R., Fernández, C. y Baptista, P. (2014). Metodología de la Investigación. 6. ed. México: McGraw-Hill.

Herrera, A. (2008). Inventario de estrategias volitivas académicas: Validación de una prueba. Tesis de Licenciatura. Universidad de las Américas, Puebla, México.

Hirmas, C. y Eroles, D. (Coords.) (2008). Convivencia democrática, inclusión y cultura de paz. Lecciones desde la práctica educativa innovadora en América Latina. Oficina Regional de Educación de la UNESCO para América Latina y el Caribe/Red Regional de Innovaciones Educativas para América Latina y el Caribe, Santiago.

Huh, J., Delorme, D., y Reid, L. (2006). Perceived third-person effects and consumer attitudes on preventing and banning DTC advertising. Journal of Consumer Affairs, 40, 90-116. 
INCLUD-ED Consortium (2012). Final INCLUD-Ed Report. Strategies for inclusion and Social Cohesion in Europe from Education. Recuperado de: http://creaub.info/included/wp-content/uploads/2010/12/D25.2_ Final-Report_final.pdf

Instituto Natura (2016). Relatorio 2016. São Paulo:AR Fernandez, Recuperado de: http://www.institutonatura.org.br/wp-content/uploads/2016/09/ Relatorio2016_InstitutoNatura_Digital-2.pdf

Jares, X. R. (2006). Pedagogía de la convivencia. Barcelona: Graó.

LLECE (2008). Los aprendizajes de los estudiantes de América Latina y el Caribe. Segundo Estudio Regional Comparativo y Explicativo (SERCE).

Lozada, J. (2014). Investigación Aplicada: Definición, Propiedad Intelectual e Industria. Cienciamérica Revista de Divulgación Científica de la Universidad Tecnológica Indoamérica, 3, 34-39.

Maldonado, H. (2004). Convivencia escolar: ensayos y experiencias. Buenos Aires: Lugar Editorial.

Marín, M. (2002). Aspectos psicosociales de la violencia en el contexto educativo. Aula Abierta, 79, 85-105.

Martín, A., Del Barrio, C. y Echeita, G. (2003). La intervención para la mejora de la convivencia en los centros educativos: modelos y ámbitos. Infancia y aprendizaje, 26, 79-95.

Martínez-Otero, V. (2001). Convivencia escolar: problemas y soluciones. Revista complutense de educación, 1, 295-318.

Mello, R. (2009). Diálogo y escuela en Brasil: comunidades de aprendizaje. Cultura y Educación, 21 (2), 171-182.

Muñoz, M., Moncada , B., Cornejo, C., Muñoz, P. \& Araya, N. (2014). Convivencia y clima escolar en una comunidad educativa inclusiva de la Provincia de Talca, Chile. Revista Electrónica de Investigación Educativa REDIE, 16(2), 16-32. Recuperado de: http://www.redalyc. org/pdf/155/15531719002.pdf 
Navareño, P. (2012). Principios para una escuela inclusiva en el siglo XXI: Avances en Supervisión Educativa. Revista de la Asociación de Inspectores de Educación de España, 16, 1-19.

Nunnally, J.C. (1967). Psychometric theory. New York: McGraw Hill.

Peralta, F., Sánchez, M, Trianes, M. y De la Fuente, J. (2003). Estudio de la validez interna y externa de un cuestionario sobre conductas problemáticas para la convivencia según el profesor. Psicología, Saúde \& Doenças, 4, 83-96. Recuperado de: http://www.redalyc.org/ pdf/362/36240106.pdf

Pulido, C. \& Zepa, B. (2010). La interpretación interactiva de los textos a través de las tertulias literarias dialógicas. Signos, 43(2), 295-309.

Red-AGE. (2014). La convivencia en los centros educativos de educación básica en Iberoamérica. Chile: Santillana.

Ramos, M.T., Ravello, C., Rivera, S., Varillas, M., Samanez, J. y Morales, S. (2013). Aprendiendo a resolver conflictos en las instituciones educativas: orientaciones para directivos y tutores de primaria $y$ secundaria. Lima, Perú: Ministerio de Educación y Ministerio de la Mujer y Poblaciones Vulnerables.

Román, M. y Murillo, J. (Agosto 2011). América Latina: violencia entre estudiantes y desempeño escolar. Revista CEPAL, 104, 37-54.

Salas, E. (2013). Diseños pre experimentales en psicología y educación: Una revisión conceptual. Liberabit revista de psicología, 19(1), 133-141.

Sánchez, H. y Reyes, C. (2006). Metodología y diseño de la investigación cientifica. Lima: Visión Universitaria.

Santos, T. (2015). Comunidades de aprendizaje y superación de la pobreza en Brasil. Intangible Capital.11 (3), 333-349.

Santos, M. y Slavin, J. (2002). La condición del éxito en la intervención pedagógica con niños en situación de riesgo: el programa Success for all. Revista de Investigación Educativa, 20(1), 173-188. Recuperado de: http://revistas.um.es/rie/ article/view/97571/93641 
UNESCO (2008). Convivencia Democrática, Inclusión y Cultura de Paz. Lecciones desde la práctica educativa innovadora en América latina. Santiago de Chile: Pehuén Editores.

Unidad de medición de la calidad (2016). ¿Cuánto aprenden nuestros estudiantes? Resultados de la ECE 2016 nacional. Lima: Ministerio de Educación

Unidad de Currículum y Evaluación (2014). Otros indicadores de calidad educativa. Santiago, Chile: Ministerio de Educación de Chile.

Vergara, M. y Ríos, J. (Coords.) (2010). Diversidad cultural: Un reto para las instituciones educativas. Guadalajara: Universidad de Guadalajara.

Vigotsky, L.S. (1996). El desarrollo de los procesos psicológicos superiores. Barcelona:Crítica.

Valls, R. (2000). Comunidades de aprendizaje: una práctica educativa de aprendizaje dialógico para la sociedad de la información. (Tesis doctoral). Universitat de Barcelona. Barcelona. Recuperado de: http:// www.tesisenred.net/handle/10803/2929

Valls, R., Prados, M. y Aguilera, A. (2014). El proyecto INCLUD-ED: estrategias para la inclusión y la cohesión social en Europa desde la educación. Revista Investigación en la Escuela, 82, 31-43.

Valls, R., Soler, M. y Flecha, R. (2008). Lectura Dialógica: Interacciones que mejoran y aceleran la lectura. Revista Iberoamericana de Educación, 47, 71-87.

Vila Merino, E. (2011). Racionalidad, diálogo y acción: Habermas y la pedagogía crítica. Revista Iberoamericana de Educación. 56(3), 1-14.

Viñas, J. (2004). Conflictos en los centros educativos. Cultura organizativa y mediación para la convivencia. Barcelona: Graó.

Wells, G. (2001). Indagación dialógica. Barcelona: Paidós 